\title{
Recommendations Regarding Corticosteroids in the Management of Multiple Sclerosis
}

\author{
a report by \\ National Clinical Advisory Board, National Multiple Sclerosis Society
}

DOI: 10.17925/USN.2008.04.01.22

While recognizing that the factors that enter into a decision to treat are complex and best analyzed by the individual patient's neurologist, the National Clinical Advisory Board of the National Multiple Sclerosis Society has adopted the following recommendations to provide guidance to clinicians, insurers, and policy-makers regarding the appropriate use of corticosteroids in the management of multiple sclerosis (MS), particularly when data are lacking or unclear. They are based on existing literature and expert opinion that is derived from clinical practice and experience. Evidence is cited where it exists, but many aspects of steroid management are not evidence-based. This article is intended as a resource for neurologists in clinical practice.

\section{Corticosteroid Treatment of Multiple Sclerosis Relapses}

Short courses of high-dose corticosteroids have been routinely used to treat acute MS relapses for many years. The first therapeutic advance in this area began with the use of adrenocorticotropic hormone (ACTH) to stimulate the synthesis of corticosteroids. ${ }^{1}$ A number of studies support the concept that steroids accelerate recovery. ${ }^{2-5}$

There is limited evidence that steroid therapy may reduce tissue damage and improve the degree of recovery, at least as determined by magnetization transfer imaging. ${ }^{6}$ However, most studies have found little difference in the ultimate degree of clinical recovery following a relapse., ${ }^{5,7}$ Despite the suggestion ${ }^{7}$ that the risk of a subsequent relapse may be reduced, other studies do not support this, nor do they support any delay in time to the next relapse. ${ }^{5,8}$ The most commonly used dosage regimen is $500-1,000 \mathrm{mg}$ of IVMP daily for three to five days, with or without a subsequent tapering dose of oral steroids for one to three weeks. ${ }^{3,9}$ Within this range, the number of days of treatment may vary depending on the type and severity of relapse and the clinician's judgment with respect to the individual patient. Although it is not clear at this time whether lower or higher doses might be more useful, 1,000mg daily can be recommended as suitable for most patients. Intravenous steroids can be administered either as a single dose per day or in divided doses. It is unclear which (if either) of these approaches is more effective. In the Optic Neuritis Treatment Trial (ONTT) ${ }^{3}$ divided dosage (250mg IVMP q6h) was used. Once-daily administration saturates steroid carriers and might lead to greater effect, but the clinical significance of these different dosing regimens is unknown. Also, the use of single or multiple infusions potentially has economic implications. Although existing data are inconclusive, the consensus of the Steroid Task Force is that a single daily dose is just as effective, more practical, and probably less costly than divided doses.

The need for an oral taper-usually of oral prednisone - and its optimal duration, if used, is uncertain. Existing data do not favor one approach over the other, and there is no real consensus as to their use. Tapers may ease the transition from taking steroids, and because of this some physicians use them routinely, but unlike the situation in systemic lupus erythematosus (SLE) it is unlikely that a taper makes any difference to overall outcome in MS. A taper should not be used if a patient previously experienced significant side effects to low-dose steroids, or if there are other reasons for withholding it, such as co-existing diabetes that might be affected adversely by steroids. Tapers have not generally been used in major clinical trials.

The question of how soon after the onset of a relapse steroid treatment should be initiated is related to two separate issues: whether to treat a specific relapse immediately and how late into a relapse it is effective to treat. Timing depends on the nature of the relapse. For example, optic neuritis is generally treated with a three-day course of steroids, as used in the ONTT, whereas more severe relapses are treated with five- or even seven-day courses. In general, we recommend that the clinician treat a major relapse (one that is potentially disabling) as soon as possible, but taking a 'wait and see' approach may be appropriate in many circumstances, especially when treating minor relapses (those that are purely sensory or cause no increase in disability). Although the steroid most often used to treat relapses is IVMP, other options may also be appropriate. For example, comparable doses of IV dexamethasone could be substituted for IVMP. Although comparative trials have not been performed, dexamethasone is a reasonable alternative if MP is unavailable, or for other reasons such as a previous allergic reaction to MP. Patients on dexamethasone may experience fewer overall side effects due to its relative lack of mineralocorticosteroid effects and consequently lower sodium retention than seen with other steroids. Dexamethasone and MP have different affinities for glucocorticoid and mineralocorticoid receptors, which may result in differences in clinical efficacy. However, such differences are likely to be minor, and MP is therefore recommended as the drug of choice.

There has recently been a renewed level of interest in using intramuscular or subcutaneous $\mathrm{ACTH}$, the original treatment for relapse management.' Although ACTH may have some theoretical advantages, it is more expensive than corticosteroids, generally has more side effects, and gives less consistent results. It may have a place in rare situations, such as when IV infusion is impractical, or in cases where its positive effects on bone via stimulation of dehydroepiandrosterone (DHEA) and mineralocorticoids may be desirable.

\section{High-dose Oral Corticosteroid Therapy}

There is evidence suggesting that oral regimens may also be effective and actually have some advantages. Studies have reported that oral therapy is equivalent to intravenous administration in other inflammatory autoimmune disorders, including asthma ${ }^{10}$ and rheumatoid arthritis.." Equivalent doses of steroids can be administered orally and intravenously, absorption and 
bioavailability are comparable, ${ }^{12}$ and the therapy is well tolerated. ${ }^{13}$ Oral therapy is more convenient for the patient and clinician, and is less expensive than traditional IV therapy. ${ }^{14}$ Furthermore, comparable oral doses may be given twice daily with food to reduce the number of tablets to be consumed with each dose. Several methods have been suggested for administering high-dose oral steroids, although none has been adequately tested. Potential regimens include a three- to seven-day course of oral methylprednisolone 1,000mg, oral dexamethasone at a dosage of $176 \mathrm{mg} /$ day, as $44 \mathrm{mg}$ tablets, or oral prednisone $1,250 \mathrm{mg} .{ }^{12}$ In the first large-scale clinical trial to address this issue, a dose of $1,400 \mathrm{mg}$ of oral MP will be compared with 1,000mg of IVMP, to allow for an estimated $70 \%$ absorption rate of the oral preparation.

Although on the basis of several relatively small studies, ${ }^{4,15}$ similar high doses of PO and IV steroids seem to have similar therapeutic benefit in treating MS relapses, it would be more reassuring if this apparent equivalence were confirmed in a larger study population. Additionally, adequate studies have not yet been performed to confirm the oral dose of each agent that is bioequivalent to $1,000 \mathrm{mg}$ IVMP. Patient reports are varied among those who were treated with both regimens, with some preferring IVMP, which is often administered at home by a nurse, and others oral therapy.

\section{Side Effects of High-dose Corticosteroids}

Short courses of high-dose corticosteroids are usually safe and reasonably well-tolerated, but they do have numerous potential adverse effects. The most common but usually not serious adverse effects associated with short courses of IV or oral corticosteroids include insomnia, dysphoria, anxiety, hyperglycemia, headache, myalgia, easy bruising, edema, palpitations, metallic taste, increased appetite, acne, flushing, and gastrointestinal distress. Although uncommon, several important adverse effects have occurred with short courses of corticosteroids, including anaphylaxis (extremely rare), various mental disturbances (e.g. manic psychosis, euphoria, or suicidal depression), avascular necrosis of bone (especially of the femoral head), hypokalemia, and gastrointestinal perforation. Exacerbations of pre-existing peptic ulcer disease, diabetes, and hypertension have also been reported. Patients in high-risk groups should be tested for potential problems related to steroid treatment. For example, diabetic patients should undergo glucose testing, patients on warfarin sodium should have their International Normalized Ratio (INR) checked, those with liver disease should be monitored, and patients with hypertension should be treated cautiously. Daily blood glucose testing is recommended as there have been rare instances in which patients without a history of diabetes have developed diabetic ketoacidosis while taking highdose steroids. There is a low risk of electrolyte imbalance depending on which steroid is used and the dose and duration of therapy. Other known metabolic abnormalities should be monitored as deemed appropriate. The incidence of problems is small in patients with relapsing-remitting MS (RRMS), most of whom are young and have few medical comorbidities. The clinician's best medical judgment should be applied on an individual basis. Repeated courses of high-dose steroids increase the risk for osteoporosis, cataracts, glaucoma, Cushingoid features, and the suppression of inflammatory and immune responses, increasing the risk for opportunistic infections. ${ }^{18}$ Even at low doses, prolonged daily steroid therapy carries similar risks and should be avoided.

\section{Low-dose Oral Prednisone-Does It Have a Place in the Therapeutic Armamentarium?}

In general, low-dose oral prednisone (approximately $1 \mathrm{mg} / \mathrm{kg}$ ) is not considered to be as effective as either high-dose oral prednisone or high-dose IVMP. For example, in optic neuritis trials high-dose IVMP seemed to produce a more rapid recovery of vision, although this was not significant at all time-points. ${ }^{3}$ This trial also reported an increased rate of recurrent optic neuritis with oral prednisone, although this observation may be spurious. ${ }^{8}$ Higher-dose therapy is used more often because there are better data demonstrating its efficacy. Nevertheless, some patients recover quickly following 10-14 days of low-dose oral therapy, and some neurologists prefer this approach in selected cases. It is, of course, impossible to determine whether an individual patient would recover from a relapse spontaneously without steroid therapy, whether high- or low-dose.
It is important for the physician to be
aware of the multiple factors that
influence decisions of when and how to
treat, and to proceed accordingly.

\section{Long-term Pulse Therapy}

Pulse therapy is often used as a single- or multiday treatment (three to five days) every few months (three to four months), or as an add-on to disease-modifying therapies at doses comparable to those used to treat relapses. In practice, it is used most often for people who are developing secondary progressive disease who have failed other therapies and before using mitoxantrone. ${ }^{19}$ Research to date has focused on whether long-term pulse IVMP therapy may delay the progression of brain atrophy or disability in patients with progressive disease. In summary:

- a five-year, phase II clinical trial of IVMP in patients with RRMS ${ }^{19}$ showed that prolonged treatment with pulsed IVMP slowed the rate of wholebrain atrophy, the development of destructive lesions (T1 black holes), and the development of sustained physical disability compared with controls;

- pulse IVMP prevented the development of brain atrophy in 11 patients with primary progressive MS (PPMS);

- a small retrospective study of patients who had received monthly pulses of IVMP showed that the treatment was associated with improvement in fatigue, spasticity, and motor strength. ${ }^{21}$ No acute exacerbations occurred in nine of 10 patients with PPMS or secondary progressive MS (SPMS);

- pulse IVMP has also been shown to prevent sustained disability. In a phase II study of 108 patients with SPMS, IVMP therapy was associated with a marginally significant delay in the onset of sustained disability; ${ }^{22}$ and

- in contrast, a single course of IVMP during an attack of acute optic neuritis failed to prevent the development of optic nerve atrophy. ${ }^{23}$

Several studies have investigated the effect of glucocorticosteroids as an add-on to standard disease management therapy, and several multicenter combination trials have either been presented in a preliminary fashion or are ongoing. A recent study ${ }^{24}$ added steroids (IVMP 1,000mg) for three successive days every other month to Avonex ${ }^{\circledR}$, and also evaluated the combination of Avonex, IVMP, and methotrexate. Data showed favorable, although not statistically significant, trends. Another double-blind controlled trial will evaluate the efficacy of interferon (IFN) $\beta-1 b$ (Betaseron ${ }^{\circledR}$ ) alone or in combination with bi-monthly IVMP in SPMS patients. The ASSERT study is 
investigating the effect of IVMP plus Copaxone ${ }^{\circledR}$ versus Copaxoner alone on brain atrophy in RRMS patients. Several studies in Europe are investigating combinations of pulse IVMP with Rebif ${ }^{\circledR}$.

Treatment with repeated IVMP pulses was not associated with osteoporosis in patients with MS who participated in a phase II trial of pulsed use of IVMP over five years. ${ }^{25}$ However, osteopenia was observed more frequently in MS patients than in healthy controls. These data suggest that repeated pulses of IVMP given over a long period do not result in a substantially increased risk for osteoporosis in MS patients. Moreover, osteopenia was found only in the control group, who had significantly higher Expanded Disability Status Scale (EDSS) scores than patients in the IVMP group, suggesting that decreased mobility may contribute to bone loss more than corticosteroid use.

\section{Steroids and Disease-modifying Therapies}

Steroids have many metabolic effects, ${ }^{26-32}$ and therefore many potential interactions and undesired side effects. The principal concern is their potential to interact with the disease-modifying agents to lower the threshold for opportunistic infections and other adverse events. However, all of the phase III pivotal trials of disease-modifying agents have permitted the use of high-dose IVMP to treat relapses, with no untoward side effects. Beta interferons synergize with steroids on magnetic resonance imaging (MRI) ${ }^{33}$ to prolong their effect on suppression of gadolinium-enhancing lesions, and high-dose steroids may be used in combination with mitoxantrone without concern about serious adverse events. ${ }^{34}$ Since steroids are immuno- suppressive and prevent the migration of immune cells to the central nervous system, there is concern about a potentially negative synergy with Tysabri ${ }^{\circledR}$. In the Atrial Fibrillation Follow-up Investigation of Rhythm Management (AFFIRM) trial, steroids were administered for acute flares and no complications of this combination were observed. Nevertheless, in clinical practice it is important to ensure, insofar as possible, that progressive multifocal leukoencephalopathy (PML) is not the cause of a suspected MS exacerbation during treatment with Tysabri. All patients receiving Tysabri must be enrolled in the FDA-mandated Tysabri Outreach: Unified Commitment to Health (TOUCH) surveillance program. In addition, an MRI scan should be considered before using steroids in this situation. Our recommendation is that steroid treatment should not be avoided in patients on disease-modifying therapies, but they should be used with care and appropriate monitoring.

\section{Summary}

Corticosteroids play an important role in the management of MS. Indications for the treatment of relapses are well established. Other indications, especially for the use of long-term intermittent pulse therapy, are less clear. Oral high-dose steroids may be considered in place of IVMP in selected patients; however, IV therapy remains the standard of care at this time. The option of low-dose oral treatment should also be retained for patients in whom this approach seems appropriate. Management may vary among patients and in the same patient at different times. It is important for the physician to be aware of the multiple factors that influence decisions of when and how to treat, and to proceed accordingly.
1. Rose AS, Kuzma JW, Kurtzke JF, et al., Co-operative study in the evaluation of therapy in multiple sclerosis: ACTH versus placebo, Final report, Neurology, 1970;20:1-58

2. Durelli L, Cocito D, Riccio A, et al., High-dose intravenous methylprednisolone in the treatment of multiple sclerosis: Clinical-immunologic correlations, Neurology, 1986;36:238-43.

3. Beck RW, Cleary PA, Anderson MM Jr, et al., A randomized, controlled trial of corticosteroids in the treatment of acute optic neuritis. The Optic Neuritis Study Group, N Engl I Med, 1992;326:581-8.

4. Sellebjerg F, Frederiksen JL, Nielsen PM, Olesen J, Double-blind, randomized, placebo-controlled study of oral, high-dose methylprednisolone in attacks of MS, Neurology, 1998;51:529-34.

5. Brusaferri F, Candelise L, Steroids for multiple sclerosis and optic neuritis, J Neurol, 2000;247:435-42.

6. Richert ND, Ostuni JL, Bash CN, et al., Interferon beta- $1 \mathrm{~b}$ and intravenous methylprednisolone promote lesion recovery in multiple sclerosis, Mult Scler, 2001;7:49-58.

7. Beck RW, Cleary PA, Trobe JD, et al., The effect of corticosteroids for acute optic neuritis on the subsequent development of multiple sclerosis, N Engl J Med, 1993;329:1764-9.

8. Goodin DS, Perils and pitfalls in the interpretation of clinical trials: A reflection on the recent experience in multiple sclerosis, Neuroepidemiol, 1999;18:53-63.

9. Barnes MP, Bateman DE, Cleland PG, et al., Intravenous methylprednisolone for multiple sclerosis in relapse, J Neurol Neurosurg Psychiatry, 1985;48:157-9.

10. Ratto D, Alfaro C, Sipsey J, et al., Are intravenous corticosteroids required in status asthmaticus?, JAMA, 1988;260:527-9.

11. Smith MD, Ahern MJ, Roberts-Thomson PJ, Pulse steroid therapy in rheumatoid arthritis, Annals of Rheumatic Diseases, 1988;47: 28-33.

12. Morrow SA, Stoian CA, Dmitrovic J, et al., The bioavailability of IV methylprednisolone and oral prednisone in multiple sclerosis, Neurology, 2004;63:1079-80.

13. Metz LM, Sabuda D, Hilsden RJ, et al., Gastric tolerance of high-dose pulse oral prednisone in multiple sclerosis, Neurology, 1999:53:2093-6.

14. Robson LS, Bain C, Beck S, et al., Cost analysis of methylprednisolone treatment of multiple sclerosis patients, Can J Neurol Sci, 1998;25:222-9.

15. Sellebjerg F, Schaldemose Nielsen H, Fredericksen JL, Olesen J, A randomized, controlled trial of oral high-dose methylprednisolone in acute optic neuritis, Neurology, 1999:52:1479-84.

16. Alam SM, Kyriakides T, Lawmen M, Newman PK, Methylprednisolone in multiple sclerosis, I Neurol Neurosurg Psychiatry, 1993;56:1219-20.

17. Barnes D, Hughes RAC, Morris RW, et al., Randomized trial of oral and intravenous methylprednisolone in acute relapses of multiple sclerosis, Lancet, 1997;349:902-6.

18. Braunwald E, Hauser S, Fauci A, et al., Harrison's 15th Edition: Principles of Internal Medicine, New York: McGraw-Hill, 2001;2: 1968.

19. Zivadinov R, Rudick RA, De Masi R, et al., Effects of IV methylprednisolone on brain atrophy in relapsing-remitting MS, Neurology, 2001;57:1239-47.

20. Pato-Pato A, Prieto JM, Lema M, et al., Cerebral atrophy in multiple sclerosis patients treated periodically with boluses of methylprednisolone, Rev Neurol, 2003;37:501-6.

21. Pirko I, Rodriguez M, Pulsed intravenous methylprednisolone therapy in progressive multiple sclerosis: Need for a controlled trial, Arch Neurol, 2004;61:1148-9.

22. Goodkin DE, Kinkel RP, Weinstock-Guttman B, et al., A phase II study of i.v. methylprednisolone in secondary-progressive multiple sclerosis, Neurology, 1998;51:239-45.

23. Hickman SJ, Kapoor R, Jones SJ, et al., Corticosteroids do not prevent optic nerve atropy following optic neuritis, JNNR, 2003;74(8):1134-41.

24. Cohen J, Calabresi P, Eickenhorst T, et al., Results of the Avonex Combination Trial (ACT). Abstract S12.005, American Academy of Neurology Annual Meeting Program, Neurology, 2007;68(12 Suppl. 1).

25. Zorzon M, Zivadinov R, Locatelli L, et al., Effects of IV methylprednisolone pulses on bone mineral density in relapsing-remitting multiple sclerosis, Eur J Neurol, 2005; 12:550-56.

26. Barkhof F, Frequin STFM, Hommes OR, et al., A correlative triad of gadolinium-DPTA MRI, EDSS, and CSF-MBP in relapsing multiple sclerosis patients treated with high-dose intravenous methylprednisolone, Neurology, 1992;42:63-7.

27. Sellebjerg F, Christiansen $M$, Jensen J, Frederiksen JL, Immunological effects of oral high-dose methylprednisolone in acute optic neuritis and multiple sclerosis, Eur I Neurol, 2000; 7:281-9.

28. Elovaara I, Lalla M, Spare E, et al., Methylprednisolone reduces adhesion molecules in blood and cerebrospinal fluid in patients with MS, Neurology, 1998;51:1703-8.

29. Gelati M, Corsini E, De Rossi M, et al., Methylprednisolone acts on peripheral blood mononuclear cells and endothelium in inhibiting migration phenomena in patients with multiple sclerosis, Arch Neurol, 2002;59:774-80.

30. Leussink VI, Jung S, Merschdorf U, et al., High-dose methylprednisolone therapy in multiple sclerosis induces apoptosis in peripheral blood lymphocytes, Arch Neurol, 2001;58:91-7.

31. Rosenberg GA, Dencoff JE, Correa N, et al., Effects of steroids on CSF matrix metalloproteinases in multiple sclerosis: Relation to blood-brain barrier injury, Neurology, 1996;46:1626-32.

32. Wandinger KP, Wessel K, Trillenberg P, et al., Effect of highdose methylprednisolone administration on immune functions in multiple sclerosis patients, Acta Neurol Scand, 1998;97: 359-65.

33. Gasperini C, Pozilli C, Bastianello S, et al., Effects of steroidson Gd-enhancing lesions before and during recombinant beta interferon 1a treatment in relapsing remitting multiple sclerosis, Neurology, 1998;50(2):403-6.

34. Edan G, Miller D, Clanet M, et al., Therapeutic effect of mitoxantrone combined with methylprednisolone in multiple sclerosis: A randomised multicentre study of active disease using MRI and clinical criteria, J Neurology Neurosurg Psych, 1997:62:112-18. 


\section{Join Us!}

SEPTEMBER 17-20, 2008

Palais des congrès de Montréal, Canada
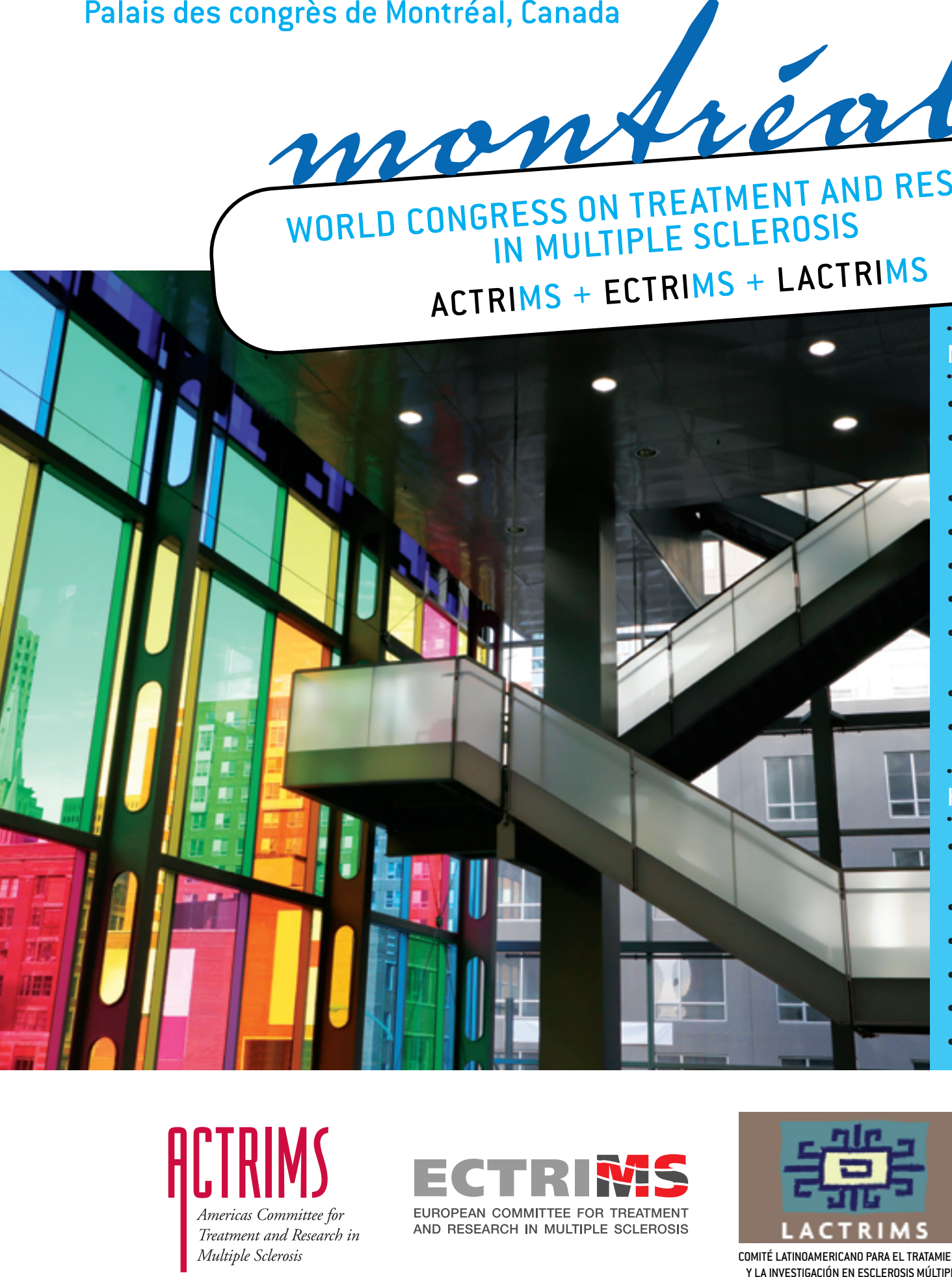

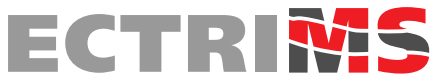

EUROPEAN COMMITTEE FOR TREATMENT AND RESEARCH IN MULTIPLE SCLEROSIS

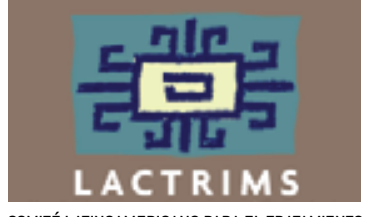

COMITÉ LATINOAMERICANO PARA EL TRATAMIENTO Y LA INVESTIGACIÓN EN ESCLEROSIS MÚLTIPLE
REGISTER TODAY! WWW.MSMONTREAL.ORG $13^{\text {TH }}$ ANNUAL MEETING OF THE AMERICAS COMMITTEE FOR TREATMENT AND RESEARCH IN MULTIPLE SCLEROSIS $24^{\text {TH }}$ CONGRESS OF THE EUROPEAN COMMITTEE FOR TREATMENT AND RESEARCH IN MULTIPLE SCLEROSIS $5^{\text {TH }}$ CONGRESS OF THE LATIN AMERICAN COMMITTEE FOR TREATMENT AND RESEARCH IN MULTIPLE SCLEROSIS
This program is offered in collaboration with the National Multiple Sclerosis Society (USA) and the Multiple Sclerosis Society of Canada. 